\title{
Structural mobility and transformations in globular proteins
}

\author{
M. VIJAYAN and DINAKAR M. SALUNKE \\ Molecular Biophysics Unit, Indian Institute of Science, Bangalore 560 012, India \\ MS received 25 June 1984
}

\begin{abstract}
Although globular proteins are endowed with well defined three-dimensional structures, they exhibit substantial mobility within the framework of the given threedimensional structure. The different types of mobility found in proteins by and large correspond to the different levels of organisational hierarchy in protein architecture. They are of considerable structural and functional significance, and can be broadly classified into (a) thermal and conformational fluctuations, (b) segmental mobility, (c) interdomain mobility and (d) intersubunit mobility. Protein crystallographic studies has provided a wealth of information on all of them. The temperature factors derived from X-ray diffraction studies provide a measure of atomic displacements caused by thermal and conformational fluctuations. The variation of displacement along the polypeptide chain have provided functionally significant information on the flexibility of different regions of the molecule in proteins such as myoglobin, lysozyme and prealbumin. Segmental mobility often involves the movement of a region or a segment of a molecule with respect to the rest, as in the transition between the apo and the holo structures of lactate dehydrogenase. It may also involve rigidification of a disordered region of the molecule as in the activation of the zymogens of serine proteases. Transitions between the apo and the holo structures of alcohol dehydrogenase, and between the free and the sugar bound forms of hexokinase, are good examples of interdomain mobility caused by hinge-bending. The capability of different domains to move semi-independently contributes greatly to the versatility of immunoglobulin molecules. Interdomain mobility in citrate synthase appears to be more complex and its study has led to an alternative description of domain closure. The classical and the most thoroughly studied case of intersubunit mobility is that in haemoglobin. The stereochemical mechanism of the action of this allosteric protein clearly brings out the functional subtilities that could be achieved through intersubunit movements. In addition to ligand binding and activation, environmental changes also often cause structural transformations. The reversible transformation between $2 \mathrm{Zn}$ insulin and $4 \mathrm{Zn}$ insulin is caused by changes in the ionic strength of the medium. Adenylate Kinase provides a good example for functionally significant reversible conformational transitions induced by variation in $\mathrm{pH}$. Available evidences indicate that reversible structural transformations in proteins could also be caused by changes in the aqueous environment, including those in the amount of water surrounding protein molecules.
\end{abstract}

Keywords. Globular proteins; conformational mobility; structural transformations; protein crystallography.

\section{Introduction}

The notion that each globular protein is endowed with a well-defined threedimensional structure has now been universally accepted primarily as a result of successful X-ray diffraction studies on protein crystals. The crystal structures of well over a hundred globular proteins are currently available. The wealth of information derived therefrom has indeed been crucial in arriving at the current ideas on the structure and action of proteins. However, each X-ray analysis, by its very nature, 
essentially gives a time and space averaged static picture of the protein. The spectacular success of protein crystallography has been such that the dynamic aspects of protein structure did not receive as much attention as they deserved, even though early protein crystallographic studies themselves had provided instances of structural changes in protein molecules (Muirhead et al., 1967; Bolton et al., 1968; Quiocho and Lipscomb, 1971). Extensive studies specifically aimed at exploring the structural mobility of proteins are thus of comparatively recent origin. Indeed, spectroscopic, particularly NMR, and theoretical studies have played a major role in developing the dynamic picture of protein molecules (Wuthrich and Wagner, 1979; Wagner, 1983; Ribeiro et al., 1982; Gurd and Rothgeb, 1979; Eftink and Ghiron, 1975; Lakowicz and Weber, 1973; Woodward et al., 1982; McCammon and Karplus, 1983; Levitt, 1983a,b), However, it turns out that a substantial part of the already available definitive information on the dynamical aspects of protein structure has been derived from protein crystallographic investigations. The focus of attention in this review is almost exclusively on the results obtained from such X-ray investigations.

A large number of crystallographic and related studies pertaining to mobility in proteins has been reported in the literature. No effort is made here to survey them exhaustively. The attempt here, perhaps the first of its kind, is to give a connected brief account, using representative examples, of the current understanding, as derived from X-ray analyses, about the mobility in proteins at different structural levels. Also discussed towards the end of the review are X-ray studies on environmental effects on protein structure with particular reference to structural transformations.

Protein molecules are highly complex structural entities involving several levels of organisational hierarchy. Mobility also therefore exists at different levels. The most common and easily understandable motions involve nonspecific rotations about single bonds in amino acid side-chains, especially those in residues occurring on the molecular surface. The other, and more significant, kinds of mobility may be classified as follows:

(i) Thermal and conformational fluctuations.

(ii) Segmental mobility.

(iii) Interdomain mobility.

(iv) Intersubunit mobility.

The different types of mobility, listed above, are by no means mutually exclusive. Indeed, almost always they coexist. It is, however, instructive to discuss them separately.

\section{Thermal and conformational fluctuations}

The X-ray diffraction pattern from a protein crystal, as indeed from any crystal, depends on the atomic positions with respect to a coordinate system defined by the unit cell and the 'temperature factors', usually denoted by $B$, of each of the atoms in the structure. The temperature factors would begin to have meaningful values only when the structure has been refined using high resolution, say better than $2 \mathrm{~A}, \mathrm{X}$-ray data. In general, each atom in a well-refined structure is thus associated with coordinates $x, y$, and $z$ which define the position of the atom, and a temperature factor $B$. The positional coordinates of all the atoms in the molecule define its structure and conformation whereas the $B$ values contain information on atomic fluctuations. 
A crystal of the size suitable for X-ray analysis contains billions of molecules distributed in nearly as many unit cells. If the molecules in all the unit cells have exactly the same structure and conformation and if they are oriented and positioned in exactly the same way, then the image of the molecule averaged over all the unit cells, which is what one obtains from X-ray crystal structure analysis, would be identical to any one molecule in the crystal. In such an ideal situation, each $B$ value, generally assumed to be isotropic in protein structures, is a direct measure, except for a constant multiplier, of the mean square amplitude of fluctuation or displacement. The time scale of thermal fluctuations is believed to be of the order of picoseconds (Gurd and Rothgeb, 1979) whereas diffraction experiments are performed over a time scale of several hours or days. Hence, what one obtains from X-ray studies are time averaged estimates of fluctuation amplitudes or atomic displacement.

Complications arise when, as is normally the case in protein crystals, all the molecules in the crystal do not have exactly the same structure and conformation. X-ray diffraction provides not only a time averaged, but also a space averaged (over all the molecules in the crystal) image. Thus if a particular atom has a position $q(x, y, z)$ in half the molecules in the crystal and $f\left(x^{\prime} y^{\prime}, z^{\prime}\right)$ in the other half, peaks at half the normal heights would appear at $\xi$ and $q^{\prime}$ in the electron density map when the two atoms are separated by a distance larger than the resolution of the map. If the separation is much smaller than the resolution, the two peaks would overlap giving rise to a single broad peak. Again, the effect of large thermal fluctuations is also to broaden the peak. The effect of static disorder (conformational fluctuations) and dynamic disorder (thermal fluctuations) cannot be separated out in any simple manner, although attempts have been made to do so by carrying out X-ray studies at different temperatures (Frauenfelder et al., 1979; Hartmann et al., 1982). Thus the amplitude of fluctuation or displacement estimated for each atom from high resolution refinement of crystal structures results from the combined effect of conformational and thermal fluctuations. Lattice disorder and translational and rotational diffusion also affect the amplitudes, but as a first approximation, these effects can either be neglected or can be assumed to be constant throughout the molecule (Frauenfelder et al., 1979). Conformational fluctuations and thermal fluctuations are indicative of flexibility, and the displacements of a group of connected atoms give a measure of the flexibility of the region of the molecule made up of these atoms.

High resolution refinement of a number of protein crystals have been carried out in recent years. The structures, the refined parameters of which have been specifically examined for fluctuations, include myoglobin (Frauenfelder et al., 1979), lysozyme (Artymiuk et al., 1979; Sternberg et al., 1979) and prealbumin (Blake and Oatley, 1982). The choice of these three proteins for discussing fluctuations is appropriate as they represent structurally and functionally different families. Myoglobin is a largely $\alpha$ helical protein and prealbumin has a $\beta$-barrel structure whereas lysozyme is made up of $\alpha$-helices as well as $\beta$-sheets $(\alpha+\beta$ structure). Myoglobin is an oxygen storage protein whereas lysozyme is a hydrolytic enzyme. The most well understood function of prealbumin is that of binding thyroid hormones.

Displacements in the three proteins, although they have very different tertiary folds, exhibit striking similarities. As is to be expected, the displacements are in general higher for the surface residues compared to those for internal residues. Also, in general, 
hydrophobic residues have lower displacements than hydrophilic residues. These two observations are of course related to a substantial extent. It has been specifically noted in the case of lysozyme and prealbumin that the molecular framework made up of sheets, helices or both have low displacements whereas loops and chain terminii are characterised by high displacements. Thus, there is a good correlation, which is eminently sensible from a structural point of view, between structure and displacement in all the three proteins considered. The variation of displacement along the polypeptide chain has also been found to be nearly the same for tetragonal hen egg white lysozyme and orthorhombic human lysozyme despite differences in crystal packing as well as the primary structure; likewise in the structure of prealbumin, variation of displacement along the polypeptide chain is similar in the two chemically identical, but crystallographically independent, subunits in the asymmetric unit. These observations and the correlation between structure and displacement referred to earlier, suggest that the observed displacements represent a molecular property rather than features resulting from crystal packing.

The displacements derived from X-ray analysis appear to have functional significance as well. For example, in myoglobin, fluctuations are low for residues in van der Waals contact with the haem group on the distal side. These residues thus form a comparatively rigid pocket for the oxygen molecule that binds to the haem iron (Frauenfelder et al., 1979). Entrance to the pocket may be possible at the distal side near residues 43-45 which have comparatively high displacement. The same path for the entrance of ligand is suggested by energy calculations as well (Case and Karplus, 1979).

An interesting feature of the displacements in the lysozyme molecule is the observed high fluctuation of the 'lips' of the active site cleft and the regions that are affected by ligand binding (figure 1). In an analogous manner, the outer part of the hormone binding site of prealbumin is also characterised by high displacements (Blake and Oatley, 1982). Presumably, high conformational flexibility of these regions is required for capturing the ligand and guiding it to the binding site. However, flexibility in the neighbourhood of the active site or the binding site does not appear to be a universal feature of protein function. In penicillopepsin, for example, the region around the active site is among those with the least mobility (James et al., 1982).

Several protein dynamics calculations have recently been carried out on structurally well characterized proteins (McCammon and Karplus, 1983). The root mean square displacements obtained from the relevant calculations are in substantial agreement with those obtained from the X-ray work referred to above (Northrup et al., 1980, 1981), thus confirming the reliability of the protein dynamics calculations and the X-ray results. Studies using techniques such as nuclear magnetic resonance (Wuthrich and Wagner, 1979; Wagner, 1983; Gurd and Rothgeb, 1979), which appears to be the method of choice for exploring fluctuations in proteins, fluorescence spectroscopy (Eftink and Ghiron, 1975; Lakowicz and Weber, 1973), solvent exchange (Woodward et al., 1982) and neutron diffraction-hydrogen exchange (Kassiakoff, 1982), also point to the fluctuating nature of protein molecules.

\section{Segmental mobility}

In many proteins, one region or segment of the molecule is more flexible than the others. This mobility often manifests itself in response to ligand binding or activation 


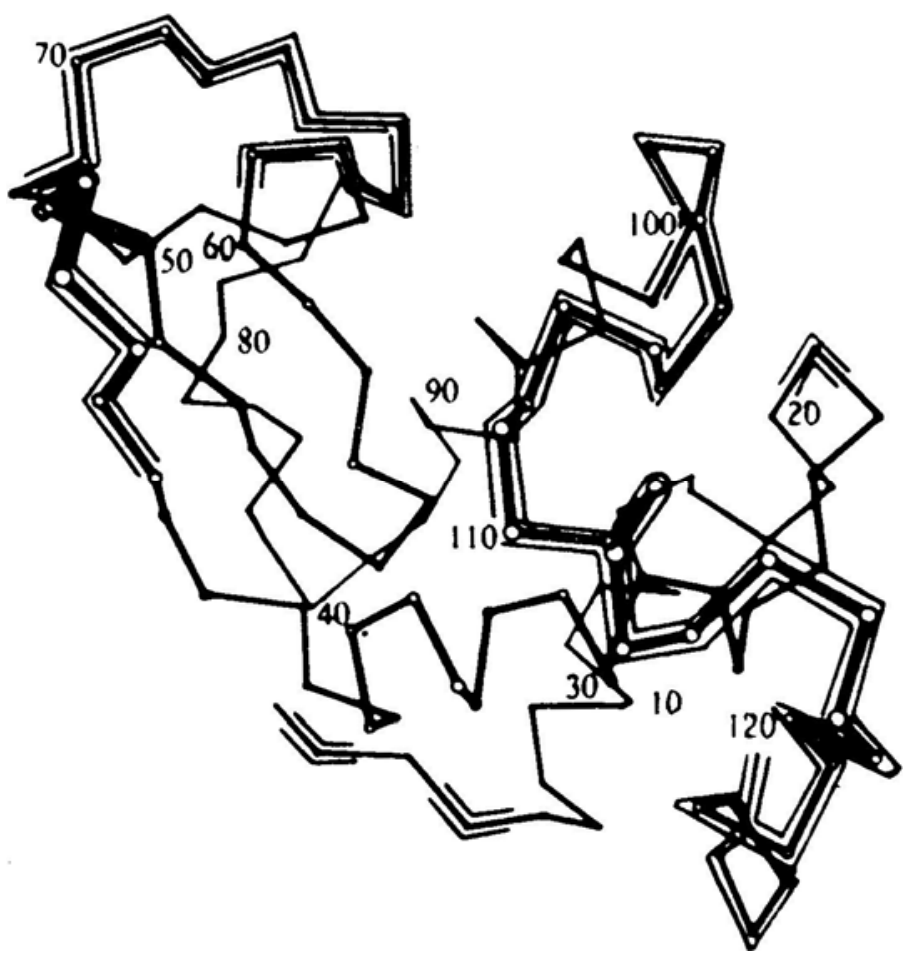

Figure 1. A perspective view of the polypeptide chain folding in lysozyme. Regions with residues having high displacement $\left(>0.2 \mathrm{~A}^{2}\right)$ in human lysozyme are indicated by the addition of two parallel lines. The high displacement of the two 'lips' of the active site cleft is clearly discernible. Reprinted by permission from Nature, 280, 563-568, Copyright (C1979, Macmillan Journals Ltd.

and has considerable functional significance. In many of the early crystallographic studies, small conformational differences, particularly those involving side chains, were observed between a free enzyme and the corresponding enzyme-inhibitor complex. The 'tyrosine-flip' in carboxypeptidase is perhaps a good example of such differences (Quiocho and Lipscomb, 1971). Much more dramatic differences, often involving a substantial segment of the polypeptide chain, were subsequently observed in many proteins.

The structure of lactate dehydrogenase provides a good example for pronounced segmental mobility involving considerable difference between the apoenzyme and its complexes (Holbrook et al., 1975). Lactate dehydrogenase is a tetrameric protein involved in the conversion of lactic acid to pyruvic acid with NAD as the coenzyme. Each subunit has two parts, viz, the coenzyme binding part and the catalytic part. As the names imply, the former binds the coenzyme whereas the substrate binding site is in the latter.

The crystal structure of the apoenzyme has been determined at $2 \mathrm{~A}$ resolution (Adams et al., 1973). Also analysed are ternary complexes with $\mathrm{NAD}^{+}$and pyruvate, $\mathrm{NADH}$ and oxamate, and $\mathrm{NAD}^{+}$and oxalate (White et al., 1976). The molecular architecture is similar in all the structures. Substantial differences in conformation, 
however, exist between the apoenzyme on the one hand and the complexes on the other. These differences can be understood by comparing, as in figure 2, the conformations of the apoenzyme and its ternary complex with $\mathrm{NAD}^{+}$and pyruvate. The greatest difference between the two structures pertains to the location, in the coenzyme binding part, of the loop region (residues 98 to 120) which includes the helix $\alpha \mathrm{D}$. The loop region, which is on the outside of the molecule, extends into the solvent in the apoenzyme. It moves closer to the main body of the molecule, covers the active centre and encloses the coenzyme and the substrate. Thus a functional active centre, protected from aqueous solvent, is produced by the loop movement.

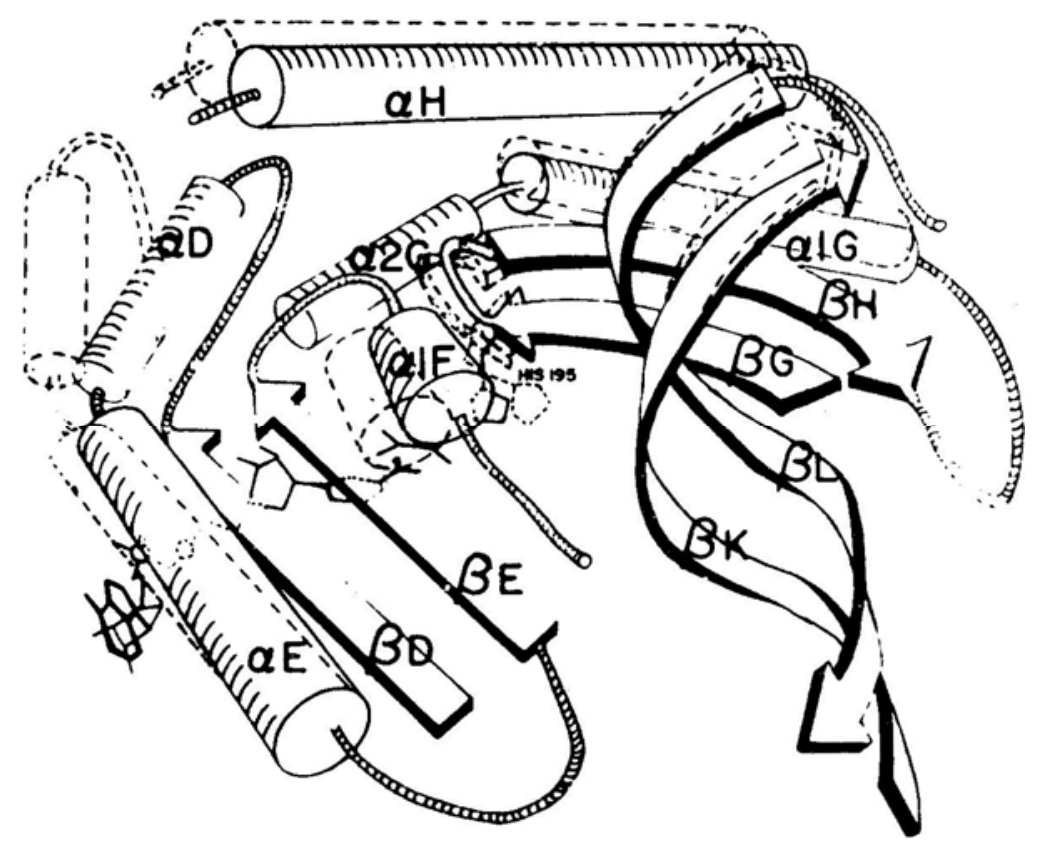

Figure 2. A representation of the differences between apo lactate dehydrogenase and its ternary complex. Conformation of the complex is drawn in unbroken lines while that of the apoenzyme is stippled. Reprinted with permission from J. Mol. Biol., 102, 759-779, Copyright (C) 1976 Academic Press (London) Ltd.

Another striking example of segmental mobility is provided in the conversion, by limited proteolysis, of zymogens of serine proteases to active enzymes. The structural basis of this conversion has been studied in the case of chymotrypsin as well as trypsin. Although the X-ray work on the chymotrypsinogen-chymotrypsin system (Sigler et al., 1968; Freer et al., 1970) was carried out earlier than that on the trypsinogen-trypsin system, we shall consider the latter in some detail as the work on this system has been more extensive and thoroughgoing (Huber and Bode, 1978). As is well known, trypsinogen is converted to trypsin by cleaving the N-terminal activation hexapeptide.

The crystal structures of pancreatic trypsin, trypsinogen and several complexes involving them have been determined at resolutions better than 2 A (Huber et al., 1974; 
Fehlhammer et al., 1977; Bode et al., 1978). The zymogen and the active enzyme have remarkably similar structures except in the region of the so called 'activation domain' made up of the N-terminal residues upto Gly-19, Gly-142 to Pro-152, Gly-184 to Gly193 and Gly-216 to Asn-223. This domain has an ordered structure in trypsin. No appreciable density exists in the region corresponding to this domain in the electrondensity map of trypsinogen indicating thereby that the peptide segments making up the domain have different conformations in different molecules or that they move about in the crystals (Fehlhammer et al., 1977). Thus, the activation domain has a relatively rigid structure in trypsin whereas it is highly flexible in trypsinogen, as illustrated in figure 3.

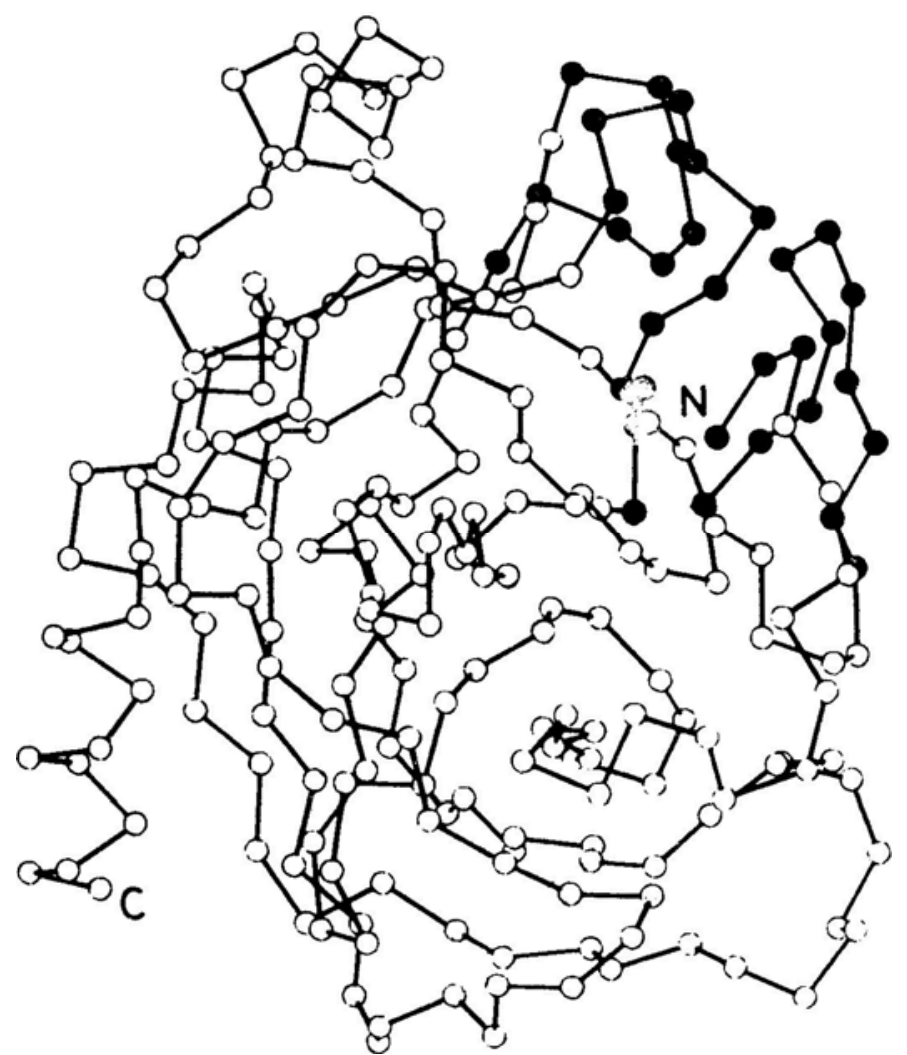

Figure 3. A representation of the structure of the trypsin molecule. Circles represent $\alpha$-carbon positions. The $\alpha$-carbon positions of residues which are disordered in trypsinogen are shown as filled circles, Atomic coordinates obtained from Cambridge Protein Data Bank ware used for preparing this figure.

The catalytic residues Asp-102, His-57 and Ser-195 have nearly the same conformation in trypsin and trypsinogen. The functional difference between the enzyme and the zymogen results from the absence of the specificity pocket in trypsinogen consequent to the flexibility of the activation domain. The rigidification of this domain and the consequent formation of the specificity pocket in trypsin is triggered by a conforma- 
tional change in Asp-194 which, on limited proteolysis, forms a salt bridge with the newly formed N-terminus of Ile-16 (Huber and Bode, 1978) .

It is interesting to note that trypsin-like conformation, with the rigidification of the activation domain, can be induced in trypsinogen even without the cleavage of the peptide bond adjacent to Ile-16. This can be achieved by complexation with a trypsin inhibitor or by the binding to the zymogen of the N-terminal dipeptide of trypsin, namely, Ile-Val, in the presence of the strong specific inhibitor $p$-guanido-benzoate (Bode et al., 1978).

A transition involving the rigidification of a flexible segment of the molecule, is by no means confined to the activation of the zymogens of serine proteases. A similar phenomenon, induced by RNA binding, occurs in tobacco mosaic virus protein (Bloomer et al., 1978; Stubbs et al., 1977) and pancreatic prophospholipase (Dijkstra et al., 1982). It appears that partial disorder in protein molecules could well have direct functional significance also. For example, it has been suggested that, the disorder, as evidenced by the absence of well-defined electron density in the Fourier map, in a part of the tyrosyl tRNA synthetase molecule may correspond to the multiple conformational states involved in different types of catalytic activity (Blow, 1978).

\section{Interdomain mobility}

Many globular proteins are made up of two or more globular autonomous substructures called domains (Wetlaufer, 1973). Lysozyme (Phillips, 1967) and papain (Drenth et al., 1968) were among the first proteins where domain structure was recognised. The active site in both these proteins is situated in the cleft between two domains. Subsequently, organisation of polypeptide chains into domains has been recognised as a basic feature of the molecular architecture of many, particularly large, proteins. For example, all the NAD dependent dehydrogenases have two domains in each of their subunits (Rossmann et al., 1974, 1975). One of them binds the coenzyme and the other the substrate with the active centre located between the domains. The coenzyme binding domain in these dehydrogenases have similar structure whereas the structures of the catalytic domain are different. Immunoglobulin provides another example of the functional significance of domain structure. It contains several domains, each associated with a specific function (Huber, 1983; Davies et al., 1975). There are also situations in which a globular protein contains two or more similar domains as in the case of $\gamma$-crystallin (Blundell et al., 1981) and wheat germ agglutinin (Wright, 1977). It has also been possible to establish similarities between domains from different proteins. These similarities have been discussed in terms of the stability of special characteristic chain foldings as well as evolutionary relationships. It has been shown (Richardson, 1981) that domains in proteins identified so far can be broadly classified into a few categories in terms of their structure and topology, suggesting the possibility of considering domains as the basic globular structural elements that go into the design of large proteins or protein subunits. The structure of domains, with particular reference to protein dynamics and function, is a topic of considerable current interest and the work pertaining to it has been reviewed recently (Janin and Wodak, 1983).

Domains in globular proteins, or protein subunits, although connected, are capable 
of some restricted independent motion. The extent of possible movement, of course, depends upon the nature of interdomain connections and vary considerably from protein to protein. Interdomain mobility, in addition to being a fascinating structural problem, is also of considerable functional significance as can be seen from the examples discussed below.

Liver alcohol dehydrogenase, an NAD dependent enzyme which oxidises alcohols to aldehydes or ketones, provides a good example for functionally important interdomain mobility (Branden et al., 1975). The enzyme, with two subunits, is a symmetric dimer. Each subunit is made up of two domains with a pocket between them. One of the domains binds the coenzyme while the other binds the substrate with the active site situated in the pocket. The catalytic zinc ion is bound to the catalytic domain well inside the pocket. The dimer formation is mediated exclusively through interactions between the coenzyme-binding domains of the two subunits. In fact the two coenzyme-binding domains together form the core of the dimeric molecule. The two catalytic domains are far apart and flank this core.

The crystal structure of the liver alcohol dehydrogenase apoenzyme and several of its binary and ternary complexes with the coenzyme or analogs and/or substrates or analogs, have been determined (Eklund et al., 1976, 1981). These structures can be broadly classified into two categories in terms of the conformation of the enzyme molecule. The first category is represented by the apoenzyme itself and in general includes complexes involving coenzyme analogs. The second category consists of most of the complexes involving the coenzyme and correspond to the productive mode of coenzyme binding. The ternary complex of the enzyme with NADH and dimethylsulphoxide represents this category. The molecular conformations in the two categories are referred to as the apoenzyme structure and the holoenzyme structure respectively. As can be seen from figure 4, which illustrates the relationship between the two structures, the transition from the apo structure to the holo structure essentially involves a rotation, about the hinge that connects the two domains, of the catalytic domain as a whole by $7 \cdot 5^{\circ}$ (Eklund et al., 1981). The transition is thus a typical case of a 'hinge bending motion'. The core of the dimeric molecule made up of the two coenzyme binding domains remains largely undisturbed except for a small movement in a short stretch in a loop region.

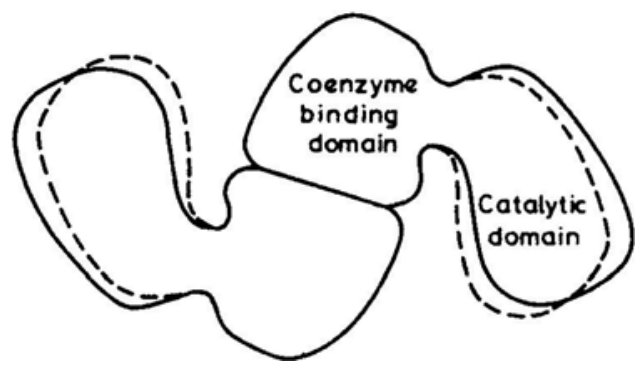

Figure 4. A schematic representation of the apo and the holo structures of the alcohol dehydrogenase dimer. Full lines and broken lines represent apo and holo structures respectively. 
The cleft in the apoenzyme is relatively open. An important consequence of the rotation of the substrate binding domain induced by coenzyme binding is for the cleft to close on the active site region between the two domains. The active site therefore is less accessible from solution and more hydrophobic in the holoenzyme. It is interesting to note that while this effect is achieved by interdomain movement, with the internal structures of the two domains largely preserved, in liver alcohol dehydrogenase, the same effect is achieved in lactate dehydrogenase, discussed in the previous section, through substantial segmental motion involving primarily a loop region in one of the domains.

Yeast hexokinase is another typical protein involving hinge-bending motion. This protein, which catalyses the phosphorylation of glucose by Mg-ATP, exists as two related isozymes, designated $A$ and $B$. The structures of several crystal forms of hexokinase and its complexes are available (Steitz et al., 1976; Anderson et al., 1978; Bennett and Steitz, 1980a,b). The enzyme molecule contains two domains, one large and the other small, with the active site in the cleft between them. The glucose binding site is in the middle of the cleft whereas ATP binds to the large domain. The molecule can exist as a dimer or a monomer. In the dimeric molecule, there is an additional ATP binding site between the two subunits.

Substantial conformational differences exist between the apoenzyme and the sugarbound enzyme. These differences, as deduced from the X-ray analysis of one of the crystal forms of the apo- $B$ isozyme and the crystals of the glucose isozyme $A$ complex, are illustrated in figure 5 . The difference can be described, to a very substantial extent, as a rotation of $12^{\circ}$ of one domain with respect to the other about an axis passing through the centre of the molecule. Whereas the cleft is relatively open in the apoenzyme, it closes on the sugar molecule in the glucose-enzyme complex. The molecule in the complex is more compact and the ligand is shielded from solvent (Bennett and Steitz, 1980b). Small angle X-ray studies in solution on isozyme $B$ and its sugar complex yield results consistent with the conformational changes on ligand-binding deduced from the single crystal studies as outlined above (McDonald et al., 1979).

The most spectacular example of interdomain mobility is perhaps found in immunoglobulin molecules. As is well known, each molecule is made up of two heavy chains and two light chains. Each heavy chain contains four domains and each light chain two domains. The domains in each chain are linked by flexible, extended polypeptide chains. The flexibility of the interdomain linkages permits the antibody molecule to assume a variety of different conformations. Many of these conformations have been defined through X-ray crystallographic studies (Huber and Bennett 1983; Amzel and Poljak, 1979). The antibody molecule has to deal with a variety of antigens and the flexibility built into its architecture is obviously designed to enable it to do so.

Yet another example of domain mobility is provided by citrate synthase which catalyses the synthesis of citrate from acetyl-coenzyme A and oxaloacetic acid. The structure of this enzyme in an open form and a closed form, in its complex with citrate and coenzyme A, has been described (Remington et al., 1982; Huber and Bennett, 1983). The enzyme has two domains, one large and one small, with a cleft in between them. The cleft is relatively open in the open form. The domains close on citrate and coenzyme A situated in the cleft in the closed form. The difference between the two forms has been explained in terms of a hinge-bending movement. Very recently, the X- 

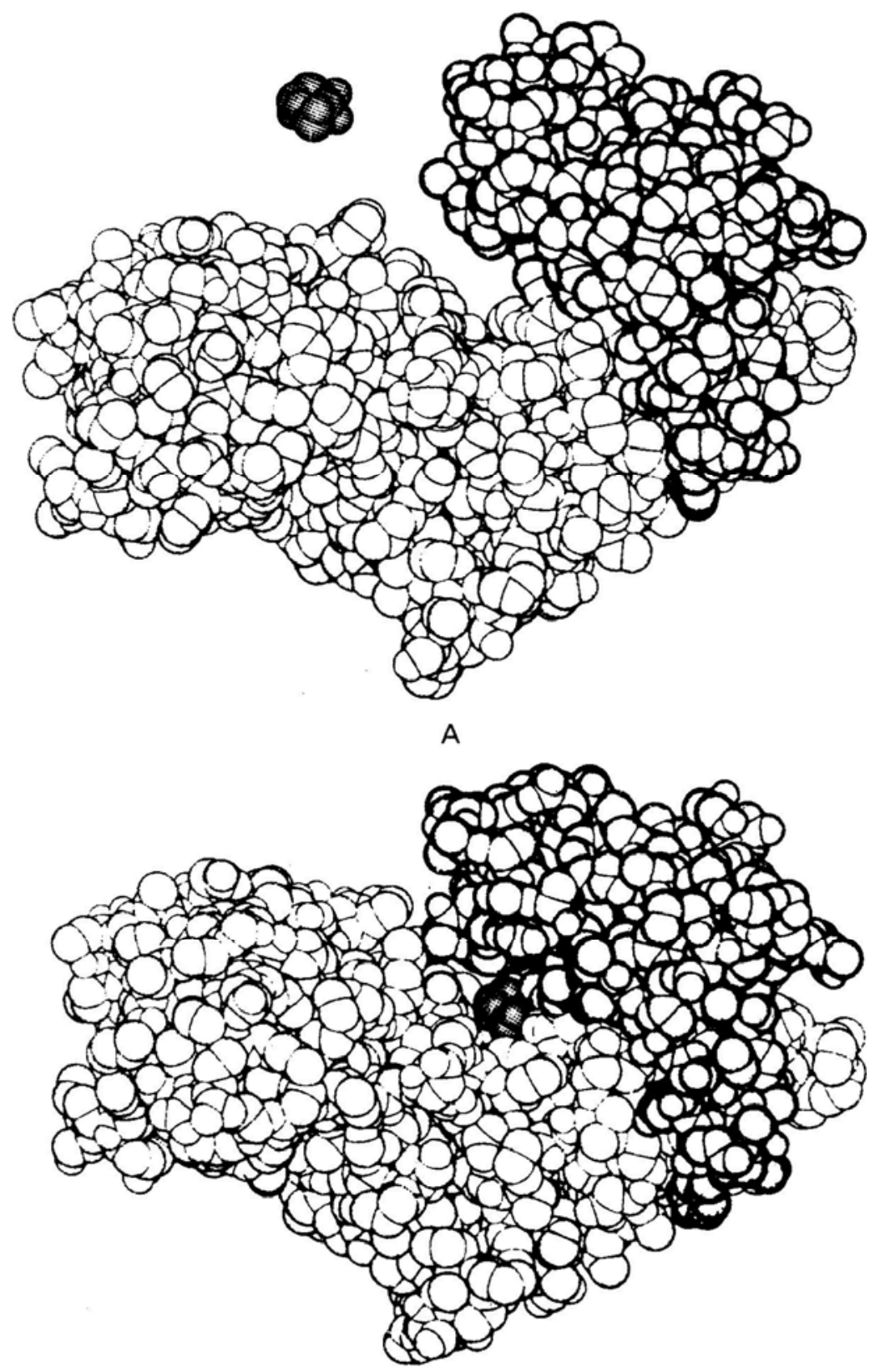

B

Figure 5. Space filling drawings of (A) free hexokinase $B$ and glucose and (B) the hexokinase $A$ : glucose complex. The small domain is more heavily shaded than the large one. Reprinted with permission from J. Mol. Biol., 140 211-230, Copyright (C) 1980, Academic Press (London) Ltd. 
ray analysis of a third crystal form of the enzyme, that of a complex with oxaloacetate formed in the presence of the inhibitor S-acetonyl-coenzyme A, has been reported (Wiegand et al., 1984). The molecules in the new form have a closed conformation. The internal structure of the two domains, particularly that of the small domain, in this form, however, deviates significantly from that observed in the open and closed forms described previously, suggesting thereby that the change from open to closed forms could be more complex than a hinge motion (Wiegand et al., 1984).

The open and closed structures of citrate synthase (Remington et al., 1982) have been recently made use of for an alternative description of domain closure (Lesk and Chothia, 1984). According to this description, domain closure, at least in citrate synthase, is caused by the cumulative effect of small shifts and rotations of packed helices. Indeed, this 'helix interface shear' mechanism and the hinge-bending mechanism are not necessarily mutually exclusive. Elements of both could coexist. For example, it has been suggested that domain closure in alcohol dehydrogenase involves features of a hinge mechanism and, in a localised form, the helix interface shear mechanism (Lesk and Chothia, 1984).

\section{Intersubunit mobility}

Almost all large proteins are oligomeric containing two or more subunits, each made up of an independent polypeptide chain. The arrangement of subunits, the quarternary structure, is generally stabilised by non-covalent interactions. The subunits in a given protein may be identical, similar or totally different. When they are identical or similar, the most frequently encountered exact or approximate symmetry of the assembly is a twofold axis or a combination of three mutually perpendicular twofold axes. Threefold, fourfold or even higher axis of symmetry between subunits is also occasionally encountered. There is at least one instance, in hexokinase, where two subunits are related by a non-integral screw operation (Steitz et al., 1976).

The classic example of intersubunit mobility is that in haemoglobin. The structure and the structural basis of action of no other protein have been studied as thoroughly as those of haemoglobin, thanks mainly to the monumental work of Perutz and his colleagues spanning over close to half a century. Many of their results are now text book material. However, the detailed mechanism of oxygen transport by haemoglobin is still a topic of extensive research. The essential features of the stereochemical basis of this mechanism, though highly complex, now appears to have been established with reasonable certainty.

As is well known, haemoglobin is a tetrameric protein made up of two $\alpha$ and two $\beta$ subunits. Each subunit carries a haem group with a central ferrous ion to which an oxygen molecule binds when the protein is oxygenated. The molecule has an exact twofold symmetry which relates the $\alpha_{1} \beta_{1}$ and $\alpha_{2} \beta_{2}$ dimers (figure 6). The two subunits within each dimer are related by an approximate twofold symmetry. Haemoglobin, unlike the closely related myoglobin, is very well known for its cooperative behaviour during ligand binding. This cooperativity is intimately connected to the quarternary structure of the molecule.

It was realised even during the low resolution X-ray analysis that unliganded (deoxy) and fully liganded (oxy) haemoglobin have somewhat different quarternary structures 


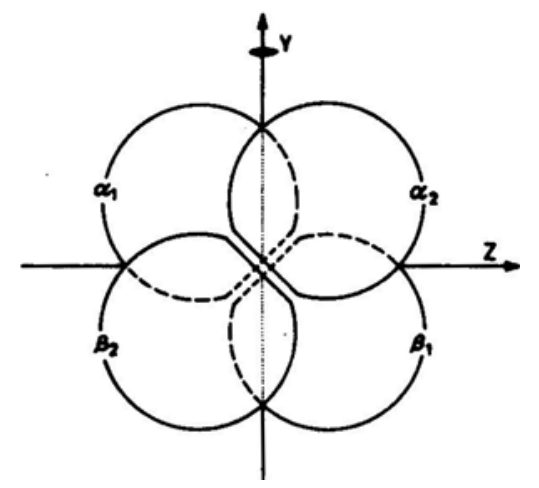

Figure 6. A schematic representation of the haemoglobin tetramer. The molecular twofold axis is along the Yaxis. Reprinted with permission from J. Mol. Biol., 129, 175-220, Copyright (C) 1979, Academic Press (London) Ltd.

(Muirhead et al., 1967; Bolton et al., 1968). On the basis of the results of the X-ray analysis of the deoxy and several liganded forms, Perutz proposed his celebrated stereochemical mechanism for the action of haemoglobin in 1970 (Perutz, 1970). This mechanism has been modified and elaborated through several investigations including high resolution X-ray analysis and theoretical studies (Baldwin, 1975; Perutz, 1979; Baldwin and Chothia, 1979; Gelin et al., 1983). This mechanism involves changes, when going from the deoxy form to the liganded form, in the tertiary structure of the subunits and the quarternary structure of the whole molecule. The changes in the tertiary and quarternary structures are indeed coupled.

The liganded or $R$ structure and the deoxy or $T$ structure have the same internal symmetry. The $T$ structure is stabilised, among other non-covalent interactions, by salt bridges involving $\mathrm{C}$-terminal residues of the subunits. These salt bridges, which have been implicated in cooperativity as well as Bohr effect, do not exist in the $R$ structure. The interactions that hold together the $\alpha \beta$ dimer, or in other words the contact region between $\alpha_{1}$ and $\beta_{1}$ (and the symmetry related $\alpha_{2}$ and $\beta_{2}$ ), remain largely unaffected during the transition between $R$ and $T$ states. The main difference between the two quarternary structures can be described as due to a rotation of the $\alpha_{2} \beta_{2}$ subunit as a whole with respect to the $\alpha_{1} \beta_{1}$ subunit. As shown in figure 7 , the axis of this rotation intersects and is perpendicular to the molecular twofold axis. It does not however pass through the molecular centre, but is situated in between the two $\alpha$ subunits at a distance of about 12 A from the centre.

Each of the two forms (deoxy and liganded) is characterised by its own tertiary and quarternary structures. The tertiary structure of one form is naturally compatible with its quarternary structure. In the deoxy form all the four ferrous ions are fivecoordinated. Four coordination sites are occupied by porphyrin nitrogens which lie in a plane. The imidazole ring from the 'proximal' histidine completes the coordination polyhedron which can be described as square pyramidal. No interaction involving the metal ion exists on the other, 'distal' side of the haem group. The ion, in this situation, is displaced from the plane of the porphyrin group towards the proximal side. The oxygen molecule when it binds to haemoglobin does so by coordinating to the haem iron at the 


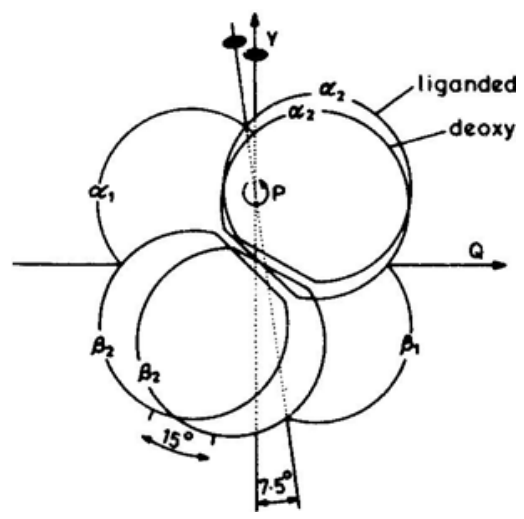

Figure 7. A schematic illustration of the quarternary structural changes in haemoglobin during the transition from the deoxy to the liganded form. $\mathrm{P}$ represents the axis about which the $\alpha_{2} \beta_{2}$ dimer rotates with respect to the $\alpha_{1} \beta 1$ dimer. Reprinted with permission from $J$. Mol. Biol., 129, 175-220, Copyright (C) 1979, Academic Press (London) Ltd.

vacant distal side. The coordination polyhedron is then octahedral, and a strong bond between the metal ion and the ligand is possible only if the ion moves closer to the haem plane. Such a movement would lead to energetically unfavourable steric strain. Hence the lower oxygen affinity of the deoxy structure.

Once a ligand attaches itself to one of the subunits in deoxyhaemoglobin, the steric strain mentioned above has to be relaxed by rearrangement in the tertiary structure in order to form a strong iron-ligand bond. These rearrangements are such as to take the tertiary structure closer to that appropriate for the $R$ structure. The atomic movements are transmitted to subunit interfaces causing a partial movement towards the $R$ quarternary structure. Now the $T$ structure is somewhat relaxed and it is easier for another ligand to bind to a second subunit. This causes a further movement towards $R$ structure making it still easier for a third ligand to bind. With the uptake of the fourth ligand, the molecule completely attains the $R$ state in which all the four ferrous ions are six coordinated with each ion located in the plane of the corresponding porphyrin nitrogen atoms.

The distances between the four binding sites (ferrous ions) in haemoglobin vary from $23 \AA$ to $39 \AA$. Ligand binding at one site however affects the affinity of the others. This is achieved not by the direct propagation of atomic movements from one site to another but by a shift in equilibrium between the $R$ and $T$ states caused by changes in the quarternary structure (Baldwin and Chothia, 1979). Indeed, haemoglobin is the best characterised allosteric protein, although the two state model outlined above differs somewhat from the one proposed by Monod and others (Perutz, 1980; Monod et al., 1965). Also, structural studies on haemoglobin clearly bring out the functional subtilities that could be achieved through intersubunit movements.

Haemoglobin, though the most thoroughly studied, is by no means the only protein in which intersubunit mobility has been characterised by X-ray methods. Another important protein and well known allosteric enzyme in which quarternary structural 
changes are currently under investigation is aspartate carbamoyl transferase (Ladner et al., 1982).

\section{Structural transformations caused by environmental effects}

Structural changes discussed in the last three sections are associated with activation or ligand binding. Striking changes in three-dimensional structure can be caused by changes in the environment such as those in ionic strength and $\mathrm{pH}$ as well. Available evidences indicate that changes in the amount and the composition of solvent around protein molecules can also lead to structural transformations.

The protein hormone insulin presents a striking case of a structural transformation due to change in the ionic strength of the medium. The tertiary structure of the hormone was first determined by the X-ray analysis of the crystals of porcine $2 \mathrm{Zn}$ insulin which contains two zinc ions per hexamer (Adams et al., 1969; Blundell et al., 1971). The three dimers in the hexamer are related to one another by an exact three-fold axis while the two monomers in each dimer are related to each other by an approximate twofold axis perpendicular to the exact threefold axis. The two zinc ions are located on the central threefold axis. One molecule from each dimer coordinate through His-B10 to one of the zinc ions while the remaining three molecules, each belonging to a different dimer, coordinate to the other.

The two molecules in the insulin dimer in $2 \mathrm{Zn}$ insulin have nearly the same conformation shown in figure 8. As is well known, pig insulin molecules contain two polypeptide chains, the 21 residue long $A$ chain and the 30 residue long $B$ chain. The two chains are held together by $A 7-B 7$ and $A 20-B 19$ disulphide bridges. In addition, there is an internal $A 6-\mathrm{A} 11$ disulphide bridge in the $A$ chain. The $A$ chain essentially contains two helices, $A 1-A 9$ and $A 12 A 19$, which are nearly antiparallel to each other. The $B$ chain has a more involved structure. It has an almost extended $\mathrm{N}$-terminal segment followed by the $B 9-B 19 \alpha$-helix; the chain then takes a ' $U$ ' turn which is followed by an extended C-terminal segment.

A different crystal form of pig insulin, $4 \mathrm{Zn}$ insulin, results when the concentration of sodium chloride in the solution is $6 \%$ or more. The repeat distances and the symmetry

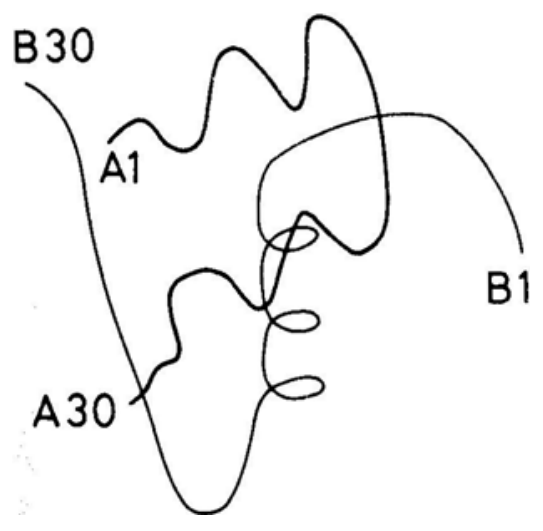

Figure 8. A schematic representation of the insulin monomer in $2 \mathrm{Zn}$ insulin. The thick and the thin lines represent the $A$ and the $B$ chains respectively. 
of these crystals are similar to those of $2 \mathrm{Zn}$ insulin. However, their X-ray analysis revealed significant changes in the internal structure (Bentley et al., 1976). $4 \mathrm{Zn}$ insulin crystals, as the name implies, contain 4 zinc ions. One of these is located on the threefold axis while the remaining three are located around it. The two molecules in the dimer are much more dissimilar to each other in $4 \mathrm{Zn}$ insulin than in $2 \mathrm{Zn}$ insulin. One of the two molecules is very similar to those in $2 \mathrm{Zn}$ insulin. The other, however, has a substantially different structure. The major difference between the two molecules pertains to the $\mathrm{N}$ terminal segment of the $B$ chain, as can be seen from figure 9. While this segment has a rather extended conformation in $2 \mathrm{Zn}$ insulin, and one of the molecules in the dimer in 4 Zn insulin, it folds and forms an N-terminal extension of the $B 9-B 19 \alpha$-helix in the other molecule in $4 \mathrm{Zn}$ insulin. This major conformational change involves atomic shifts upto about $20 \mathrm{~A}$. It also leads to changes in zinc coordination and in the rest of the molecule. The functional significance of these changes, as indeed the molecular mechanism of insulin action, is still unknown. In any case, it is remarkable that large changes of the type observed in $4 \mathrm{Zn}$ insulin could be brought about, that too reversibly (Bentley et al., 1978), exclusively by changing the ionic strength of the medium.

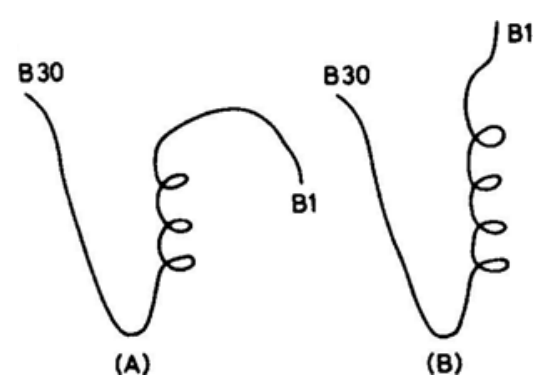

Figure 9. Schematic representations of the $B$ chain in (A) the molecules in $2 \mathrm{Zn}$ insulin and one of the monomers in the dimer of $4 \mathrm{Zn}$ insulin and (B) the other monomer in the dimer of $4 \mathrm{Zn}$ insulin.

Adenylate kinase provides a good example for conformational transformations caused by variation of $\mathrm{pH}$. This comparatively small enzyme catalyses the reaction

$$
\mathrm{Mg} \mathrm{ATP}+\mathrm{AMP} \rightleftharpoons \mathrm{Mg} \mathrm{ADP}+\mathrm{ADP} .
$$

The enzyme crystallizes in three different, though related, forms depending upon the $\mathrm{pH}$ of the medium. Of these three, the X-ray analysis of two forms, designated $A$ and $B$, has been carried out (Schulz et al., 1974; Sachsenheimer and Schulz, 1977). $A$ form crystallizes in the $\mathrm{pH}$ range $6 \cdot 9-8 \cdot 0$ and $B$ form in the range $5 \cdot 7-5 \cdot 9$, with a hysteresis range of 5.9 to 6.9 . A pre-grown crystal of one form can be transformed to the other by appropriately changing the $\mathrm{pH}$ of the mother liquor surrounding the crystal.

The overall tertiary fold of the molecule is very similar in $A$ and $B$. However, as illustrated in figure 10, substantial conformational difference, involving $15 \%$ of the residues, exists between the two forms. The largest change, about $6 \mathrm{~A}$, occurs in the loop between residues 16 and 22. This change, along with the tilt in the 23-30 helix, opens up a hydrophobic pocket formed by the central $\beta$-sheet, the C-terminal helix and 23-30 helix, in the transition from $A$ to $B$. Helix 123-133 moves away from the centre of the molecule presumably to accommodate the loop 16-23 in its changed position in $B$. Other, smaller changes, indicated in figure 10, also occur during the transition. On the basis of a close examination of the crystal structures and other lines of evidence, the 


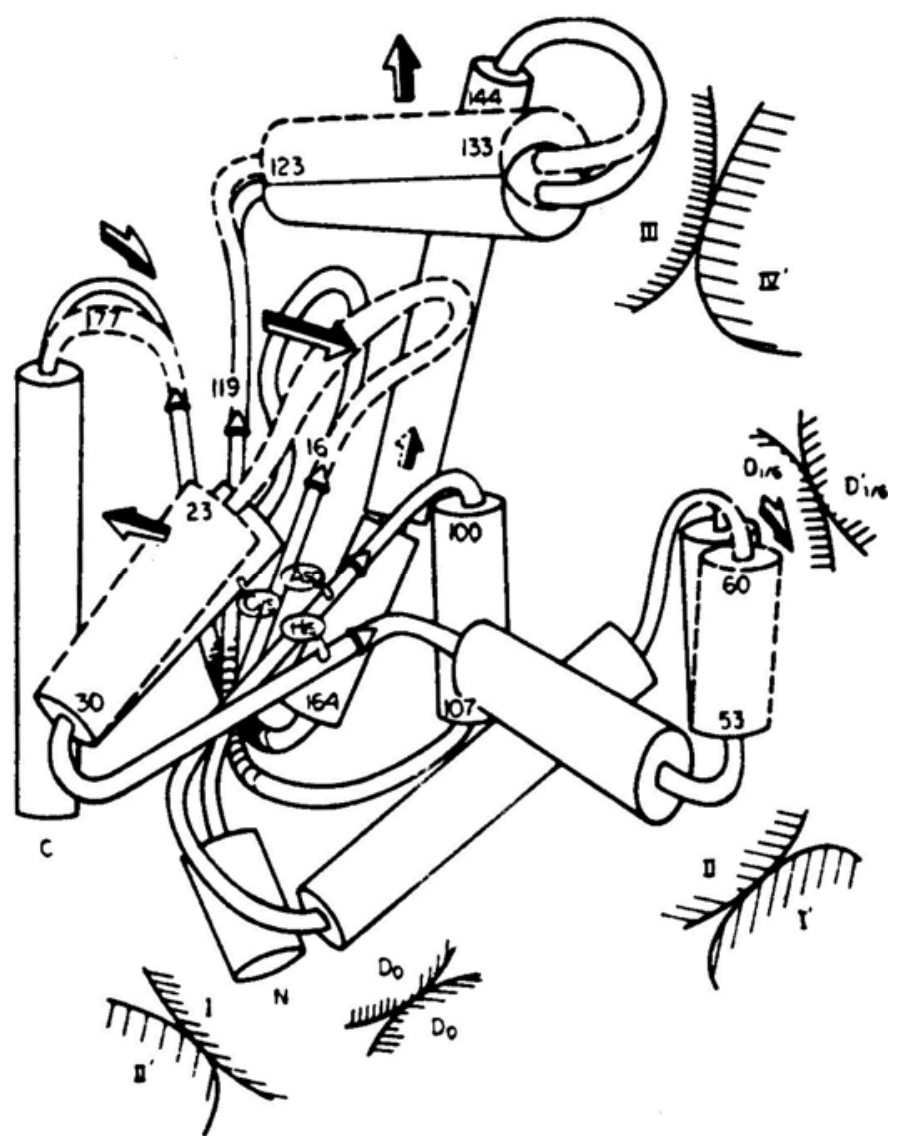

Figure 10. A representation of the $A$ (solid lines) and $B$ (broken lines) conformations of adenylate kinase. Reprinted with permission from J. Mol. Biol., 114, 23-26, Copyright (C) 1977 , Academic Press (London) Ltd.

conformational changes that accompany the transition between $A$ and $B$, have been suggested to be an intrinsic molecular property rather than the effect of changes in crystal packing. The midpoint of the $\mathrm{pH}$ range of hysteresis in $A \rightarrow B$ and $B \rightarrow A$ transitions is very close to the pK value of His-36 located at the entrance of the hydrophobic pocket referred to earlier. The change in the ionisation state of this residue has been suggested to be the triggering mechanism for the $A \rightleftharpoons B$ transition (Sachsenheimer and Schulz, 1977).

The two conformational states outlined above have been suggested to be related to the induced fit mechanism of enzyme action (Pai et al., 1977). Conformation $B$ is identified with the free enzyme $E$ and $A$ with $E^{*}$, the enzyme species after the substrate induced conformational change. The epzyme has binding sites for ATP as well as AMP. The hydrophobic pocket, referred to earlier, has been identified as the binding site for adenosine in AMP. According to this model, the substrates, particularly AMP, trigger the switch between $A$ and $B$. His-36 located at the entrance of the AMP site, is naturally 
affected by substrate binding. In the crystal form $A$, the sulphate ions bound to the enzyme mimic the action of phosphates in the substrates and this explains the existence of the conformation appropriate for $E^{*}$ in these crystals even in the absence of substrates.

It appears that structural transformations in protein crystals could be caused by changes in the aqueous environment as well. The earliest experiments bearing on this problem were conducted way back in the late forties and the early fifties when the crystals of haemoglobin were examined under varying conditions of environmental humidity and salt concentration in the mother liquor (Boyes-Watson et al., 1947; Bragg and Perutz, 1952; Huxley and Kendrew, 1953). The results were then explained as due to the movement of molecular layers. The ramifications of the results were not further explored at that time, which is not surprising as protein crystallography was then at its infancy.

Recent experiments in our laboratory on water mediated structural transformations caused by changes in the relative humidity of the environment, suggest that such transformations could well occur in a variety of protein crystals. The crystals on which systematic studies of this type have been carried out include a new crystal form of ribonuclease $A$ and the well known tetragonal lysozyme (Salunke et al., 1984). As illustrated in figure 11, the unit cell volume of the new form of ribonuclease $A$ reduces discontinuously between 95 and $93 \%$ relative humidity when the humidity of the environment of the crystal is reduced systematically. As is well known, all protein crystals contain a substantial amount of solvent which surrounds the protein molecules. In the case of the new form of ribonuclease $A, 41 \%$ of the crystal is made up of aqueous solvent. The discontinuous change in unit cell volume, illustrated in the figure 11 , corresponds to a reduction in solvent content to $37 \%$ of the crystal volume. This reduction in volume is accompanied by significant changes in the diffraction pattern presumably caused by changes in molecular conformation as well as crystal

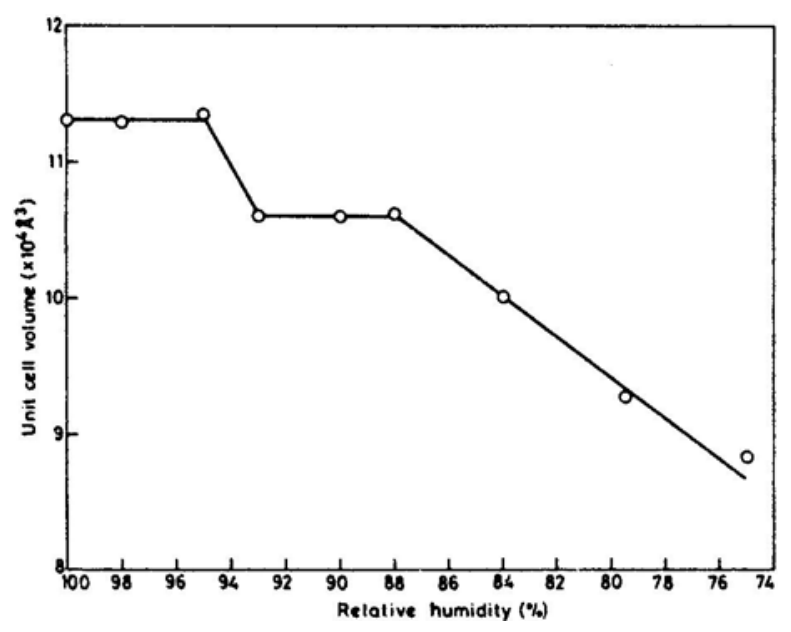

Figure 11. Variation of the unit cell volume in the new crystal form of ribonuclease $A$ as a function of the relative humidity of the environment. 
packing. Studies on tetragonal lysozyme also yield results similar to those obtained in the case of ribonuclease $A$. In both the cases, the transformation is reversible in terms of unit cell dimensions and the diffraction pattern. It turns out that this water-mediated transformation is affected by the composition of the solvent that surrounds the crystals. For example, 2-methylpentan 2,4-diol (MPD), when used as a cosolvent, reduces the relative humidity at which the transformation takes place in the new form of ribonuclease $A$.

Proteins almost always exist and function in aqueous environment. Indeed, hydration of proteins and the effects connected with it have received considerable attention (Careri et al., 1980; Baker et al., 1983; Poole and Finney, 1983; Kuntz and Kauzmann, 1974). In view of the importance of water in the structure and action of proteins, changes in aqueous environment could conceivably have profound effects on protein conformation. Water-mediated transformations provide a useful means for exploring these effects.

\section{Acknowledgements}

The authors thank Dr. M. R. N. Murthy for many useful discussions. Their thanks are also due to SERC, Department of Science and Technology, New Delhi for financial support.

\section{References}

Adams, M. J., Blundell, T. L., Dodson, E. J., Dodson, G. G., Vijayan, M, Baker, E. N., Harding, M. M., Hodgkin, D. G, Rimmer, B. and Sheat, S. (1969) Nature (London), 224, 491.

Adams, M. J., Ford, G. C, Liljas, A. and Rossmann, M. G. (1973) Biochem. Biophys. Res. Commun., 53, 46. Amzel, L. M. and Poljack, R. J. (1979) Ann. Rev. Biochem., 48, 961.

Anderson, C. M., Stenkamp, R. E. and Steitz, T. A. (1978) J. Mol. Biol., 123, 15.

Artymiuk, P. J., Blake, C. C. F., Grace, D. E. P., Oatley, S. J., Phillips, D. C. and Sternberg, M. J. E. (1979) Nature (London), 280, 563.

Baker, L. J., Hansen, A. M. F., Bhaskara Rao, P. and Bryan, W. P. (1983) Biopolymers, 22, 1637.

Baldwin, J. M. (1975) Prog. Biophys. Mol. Biol., 29, 225.

Baldwin, J. and Chothia, C. (1979) J. Mol. Biol., 129, 175.

Bennett, W. S. Jr. and Steitz, T. A. (1980a) J. Mol. Biol., 140, 183.

Bennett, W. S. Jr. and Steitz, T. A. (1980b) J. Mol. Biol., 140, 211.

Bentley, G., Dodson, E., Dodson, G., Hodgkin, D. and Mercola, D. (1976) Nature (London), 261, 166.

Bentley, G., Dodson, G. and Levitova, A. (1978) J. Mol. Biol., 126, 871.

Blake, C. C. F. and Oatley, S. J. (1982) in Conformation in Biology (eds. R. Srinivasan and R. H. Sarma) (New York: Adenine Press), p. 29.

Bloomer, A. C, Champness, J. N., Bricogne, G., Staden, R. and Klug, A. (1978) Nature (London), $276,362$.

Blow, D. M. (1978) in Molecular Interactions and Activity in Proteins, Ciba Foundation Symposium-60 (New Ser.), (Amsterdam: Excerpta Medica and New York: North-Holland Inc.) p. 55.

Blundell, T. L., Cutfield, J. F., Cutfield, S. M., Dodson, E. J., Dodson, G. G., Hodgkin, D. C, Mercola, D. A. and Vijayan, M. (1971) Nature (London), 231, 506.

Blundell, T., Lindley, P., Miller, L., Moss, D., Slingsby, C., Tickle, I., Turnell, B. and Wistow, B. (1981) Nature (London), 289, 771.

Bode, W., Schwager, P. and Huber, R. (1978) J. Mol. Biol., 118, 99.

Bolton, W., Cox, J. M. and Perutz, M. F. (1968) J. Mol. Biol., 33, 283.

Boyes-Watson, J., Davidson, E. and Perutz, M. F. (1947) Proc. R. Soc. (London), A191, 83. 
Bragg, L. and Perutz, M. F. (1952) Proc. R. Soc. (London), A213, 425.

Branden, C. I., Jornvall, H, Eklund, H. and Furugren, B. (1975) in The Enzymes (ed. P. D. Boyer) 3rd edition, Vol. 11, p. 103.

Careri, G., Grotton, E., Yong, P. H. and Rupley, J. A. (1980) Nature (London), 284, 572.

Case, D. A. and Karplus, M. (1979) J. Mol. Biol. 132, 343.

Davies, D. R., Padlan, E. A. and Segal, D. M. (1975) Ann. Rev. Biochem., 44, 639.

Dijkstra, B. W., VanNes, G. J. H., Kalk, K. H., Brandenburg, N. P., Hol, W. G. J. and Drenth, J. (1982) Acta Crystallogr., B38, 793.

Drenth, J., Jansonius, N., Koekoek, R., Swen, H. and Wolthers, B. (1968) Nature (London), 218, 929. Eftink, M. R. and Ghiron, C. A. (1975) Proc. Nat. Acad. Sci. USA, 72, 3290.

Eklund, H., Nordstrom, B., Zeppezauer, E., Sodurlund, G., Ohlsonn, I., Boiwe, T., Soderberg, B. O., Tapia, O., Branden, C. I. and Akesson, A. (1976) J. Mol. Biol., 102, 27.

Eklund, H., Samama, J. P., Wallen, L., Branden, C. I., Akesson, A. and Jones, T. A. (1981) J. Mol. Biol., 146, 561.

Fehlhammer, H., Bode, W. and Huber, R. (1977) J. Mol. Biol., 111, 415.

Frauenfelder, H., Petsko, G. A. and Tsernoglou, D. (1979) Nature (London), 280, 558.

Freer, S. T., Kraut, J., Robertus, J. D., Wright, H. T. and Xuang, Ng. H. (1970) Biochemistry, 9, 1997. Gelin, B. R., Lee, A. W. M. and Karplus, M. (1983) J. Mol. Biol., 171, 489.

Gurd, F. R. N. and Rothgeb, T. M. (1979) Adv. Protein Chem., 33, 73.

Hartmann, H., Parak, F., Steigemann, W., Petsko, G. A., Ringe Ponzi, D. and Frauenfelder, H. (1982) Proc. Natl. Acad. Sci. USA, 79, 4967.

Holbrook, J. J., Liljas, A., Steindel, S. J. and Rossmann, M. G. (1975) in The Enzymes (ed. P. D. Boyer) 3rd edition, Vol. 11, p. 191.

Huber, R. and Bennett, W. S. (1983) Biopolymers, 22, 261.

Huber, R. and Bode, W. (1978) Acc. Chem. Res., 11, 114.

Huber, R., Kukla, D., Bode, W., Schwager, P., Bartels, K., Deisenhofer, J. and Steigemann, W. (1974) J. Mol. Biol., 89, 73.

Huxley, H. E. and Kendrew, J. C. (1953) Acta Crystallogr. 6, 76.

James, M. N. G., Seilecki, A., Salituro, F., Rich, D. H. and Hofmann, T. (1982) Proc. Natl. Acad. Sci. USA, 79, 6137.

Janin, J. and Wodak, S. J. (1983) Prog. Biophys. Mol. Biol., 42, 21.

Kassiakoff, A. A. (1982) Nature (London), 296, 713.

Kuntz, Jr. I. D. and Kauzmann, W. (1974) Adv. Protein Chem., 28, 239.

Ladner, J. E., Kitchell, J. P., Honzotko, H. M. Ke., Volz, K. W., Kolb (Gilboa), A. J., Ladner, R. C. and Lipscomb, W. N. (1982) Proc. Natl Acad. Sci. USA, 79, 3125.

Lakowicz, J. R. and Weber, G. (1973) Biochemistry, 12, 4171.

Lesk, A. M. and Chothia, C. (1984) J. Mol. Biol., 174, 175.

Levitt, M. (1983a) J. Mol. Biol., 168, 595.

Levitt, M. (1983b) J. Mol. Biol., 168, 621.

McCammon, J. A. and Karplus, M. (1983) Acc. Chem. Res. 16, 187.

McDonald, R. C, Steitz, T. A. and Engelman, D. M. (1979) Biochemistry, 18, 338.

Monod, J., Wyman, J. and Changeux, J. F. (1965) J. Mol. Biol., 12, 88.

Muirhead, H., Cox, J. M., Mazzarella, L. and Perutz, M. F. (1967) J. Mol. Biol., 28, 117.

Northrup, S. H., pear, M. R., McCammon, J. A. and Karplus, M. (1981) J. Mol. Biol, 153, 1087.

Northrup, S. H., Pear, .. R., McCammon, J. A., Karplus, M. and Takano, T. (1980) Nature (London), 287, 659.

Pai, E. F., Sachsenheimer, W., Schirmer, R. H. and Schulz, G. E. (1977) J. Mol. Biol., 144, 37.

Perutz, .. F. (1970) Nature (London), 228, 726.

Perutz, M. F. (1979) Ann. Rev. Biochem., 48, 327.

Perutz, M. F. (1980) Proc. R. Soc. (London), B208, 135.

Phillips, D. C. (1967) Proc. Natl. Acad. Sci. USA, 57, 484.

Poole, P. L. and Finney, J. L. (1983) Biopolymers, 22, 255.

Quiocho, F. A. and Lipscomb, W. N. (1971) Adv. Protein Chem., 25, 1.

Remington, S., Weigand, G. and Huber, R. (1982) J. Mol. Biol., 158, 11. 
Ribeiro, A. A., King, R. and Jardetzky, O. (1982) in Conformation in Biology (eds R. Srinivasan and R. H. Sarma) (New York: Adenine Press), p. 39.

Richardson, J. (1981) Adv. Protein Chem., 34, 167.

Rossmann, M. G., Liljas, A., Branden, C. I. and Banaszak, L. J. (1975) in The Enzymes, ed. P. D. Boyer, 3rd edition, Vol. 11, p. 61.

Rossmann, M. G., Moras, D. and Olsen, K. W. (1974) Nature (London), 205, 194.

Sachsenheimer, W. and Schulz, G. E. (1977) J. Mol. Biol., 144, 23.

Salunke, D. M., Veerapandian, B. and Vijayan, M. (1984) Curr. Sci., 53, 231.

Schulz, G. E., Elzinga, M, Marx, F. and Schirmer, R. H. (1974) Nature (London), 250, 120.

Sigler, P. B., Blow, D. M., Matthews, B. W. and Henderson, R. (1968) J. Mol. Biol., 35, 143.

Steitz, T. A., Fletterick, R. J., Anderson, W. F. and Anderson, C. M. (1976) J. Mol. Biol., 104, 197.

Sternberg, M. J. E., Grace, D. E. P. and Phillips, D. C (1979) J. Mol. Biol., 130, 231.

Stubbs, G., Warren, S. and Holmes, K. (1977) Nature (London), 267, 216.

Wagner, G. (1983) Q. Rev. Biophys., 16, 1.

Wiegand, G., Remington, S., Deisenhofer, J. and Huber, R. (1984) J. Mol. Biol., 174, 205.

Wetlaufer, D. B. (1973) Proc. Nat. Acad. Sci. (USA), 70, 697.

White, J. L, Hackert, M. L, Buehner, M., Adams, M. J., Ford, G. C, Lentz, Jr. P. J., Smiley, I. E., Steindel, S. J. and Rossmann, M. G. (1976) J. Mol. Biol., 102, 759.

Woodward, C, Simon, I. and Tuchsen, E. (1982) Mol. Cell. Biochem., 48, 135.

Wright, C. S. (1977) J. Mol. Biol., 111, 439.

Wuthrich, K. and Wagner, G. (1979) Trends Biochem. Sci., 3, 227. 